\title{
On the Provision of Advanced Telecommunication Services in Rural Areas
}

\author{
Lambros Lambrinos \\ Department of Communication and Internet Studies, Cyprus University of Technology \\ lambros. lambrinos@cut.ac.cy
}

\begin{abstract}
Even though in many parts of the world people enjoy the features of modern telecommunication services, a significant number of inhabitants in rural areas are deprived of access to such facilities. In this text we propose how we can utilize existing technologies for the provision of telecommunication facilities in rural areas: IP telephony based upon open source software and offthe-shelf hardware and voice messaging based upon the Delay Tolerant Networking paradigm.
\end{abstract}

Keywords: VoIP, delay tolerant network, voicemail, rural telecommunications.

\section{Introduction}

In the current era people take advanced telecommunication services for granted and assume that such facilities are available worldwide. Telecommunication operators are not prepared to invest in areas that are not profitable for them with prime examples being rural places with a small number of inhabitants or places that are remote and infrastructure development costs are high. As a result, there are many areas in the world lacking access to the latest telephony and internet technologies and when those technologies are provided, their cost may not be affordable for most of the potential users residing in such areas due to socio-economic factors.

In the context of this work, there is particular interest in dealing with two scenarios: in the first, there exist many situations where advanced telephony (e.g. voice-mail) and internet services are available in an area but their cost may be prohibitively high for the potential customers; in the second, there are areas that are totally disconnected and no telephony and internet services are provided at all or they are provided opportunistically and with limited functionality.

\section{Advanced Telephony Features for Rural Areas}

Evidently, rural areas do not always enjoy the advanced telecommunications facilities that are available in urban settings. However, during the last few years, the landscape in the telecommunications sector is changing dramatically; the large and traditional telecom operators are no longer monopolizing the market and face fierce competition from alternative carriers and cable operators. The major reason for this being data 
convergence: the conversion of speech and video signals into data which is 'transported' using the same networks used for traditional Internet traffic (for example, email, web browsing and file exchange data). Data convergence has resulted in radical changes in the telecommunications sector and has enriched Internet-based communication between users.

Our goal is to demonstrate how community-driven efforts can assist in reducing the cost of telecommunications, as well as, in providing access to features that would have not been available otherwise. With such an observation at hand, we propose here the design of a low-cost wireless network architecture (Figure 1) to support voice services over Internet based networks, i.e. develop a VoIP infrastructure for rural areas. To minimize costs, we chose to use freely available open-source soft-ware in the core of our system as explained in the sequel.

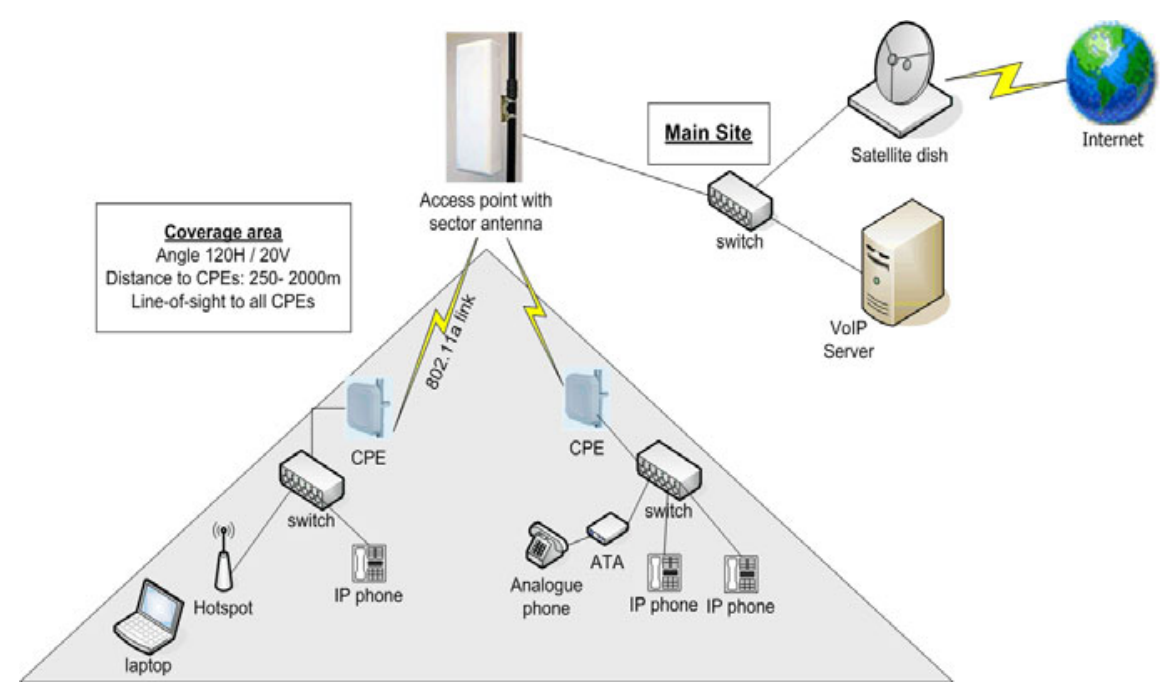

Fig. 1. A VoIP telephony implementation for rural areas

Voice and video over an IP network provide for an enriched and low-cost communication experience. Initial implementations of VoIP technology were based on proprietary protocols which gradually gave way to more open interoperability standards with the Session Initiation Protocol (SIP) [8] being widely adopted. SIP is a flexible text-based application layer signaling protocol used in the setup and termination of multimedia sessions (e.g. VoIP calls); it follows a request-response approach in its operation.

Current applications of SIP may operate based upon the peer-to-peer model (i.e. there is direct SIP-based communication between two entities) or may rely upon a SIP server which acts as a registrar holding location information and preferences for its registered users; such information is used for call establishment. As a result of its wide appeal, many end user devices (IP phones) as well as software tools (soft phones) implement SIP; along with a range of SIP compliant IP-PBXs they constitute a fully fledged telephony solution. The IP-PBX systems support a vast number of 
features that range from simple voice messaging to Interactive Voice Response (IVR), Conference Calling, and Automatic Call Distribution.

Our system is designed to provide enhanced telephony services in a rural community and it is based upon an open source implementation of a SIP PBX (asterisk [1]) and other low-cost technologies. Using a wireless infrastructure we interconnect our SIP-based telephony exchange with the client endpoints installed at various locations enabling seamless internal communication as well as connectivity to the normal telephone network (facilitated over analogue lines and a satellite link).

Wireless networks offer rapid, and hence low cost, connectivity solutions in scenarios where wired networks are not easily implementable; as discussed this is exactly the case in rural areas. The continuous enhancements in terms of bandwidth and link distance enable the deployment of long distance (tens of kilometers) point-topoint links between two locations in addition to the traditional hotspot deployments.

The pilot implementation of the architecture has provided some interesting findings and pinpointed some open issues. An open source IP-PBX was used and the endpoints ranged from simple and advanced IP phones, adaptors for existing analogue phones to preserve user device familiarity as well as software phones (soft phones). Users welcomed the extra features provided in comparison with the plain analogue telephony they were used to; more details can be found in [9].

\section{$3 \quad$ Utilizing Delay Tolerant Networks for Voice Messaging}

The scenario presented in the previous section assumed that services are generally available in an area; some areas in the world do not even have a basic telephone connection and long range wireless links are used to provide rudimentary access (e.g. through a single location servicing a whole village) to the Public Switched Telephone Network (PSTN) and perhaps the Internet.

One other feature that is still quite popular in the analogue and mobile telephony world is voice messaging which is also offered by VoIP-based (i.e. packet switched) systems; one advantage is that such systems can also be configured to send the message as an email attachment to the user associated with the number called. Voicemail-to-email is primarily done in order to expedite delivery of the message (e.g. the user is not at work but has email access) but the user's email may also not be readily accessible.

Since voice messaging is by nature delay tolerant, it is well suited in scenarios where connectivity is intermittent or network resources are scarce and real-time voice communications can not be guaranteed. To realize the envisioned elastic voice messaging implementation we suggest the deployment of local infrastructures in combination with Delay Tolerant Networks (DTN) for data transfers. More particularly, we look into the provision of voicemail facilities over DTN; such a service allows users in an area where a telephony service is not regularly available (or not available at all) to communicate (through voice messages) with users connected to the PSTN.

Note, that our target application is slightly different than traditional voice messaging where message delivery takes place immediately and access time depends 
solely on the recipient; we have another inherent delay due to the frequent delivery network disconnectivity but on the other hand, a messaging service is provided where it would not normally be available.

Evidently to support such delay-tolerant messaging service, traditional TCP/IP protocols could not suffice as the viable approach. Instead, DTN techniques are employed that are able to combat disconnections and sustain information flow. A DTN defines a network overlay architecture [3] that delivers packets using asynchronous message forwarding. It is designed to operate in environments with limited expectations of end-to-end connectivity and node resources; to this extent, it was initially proposed to facilitate interplanetary communication but as we will see it has also been useful in other applications.

Briefly put, DTNs are based upon a store-and-forward approach for data delivery. Nodes within a DTN network act as mules; they store received messages and hold them until they "carry" them over to the next hop on their way to their destination. Before leaving its source, data is assembled into 'bundles'; the bundle protocol [2] is an application layer protocol that manages bundle propagation with the DTN area.

To interconnect a DTN with the internet infrastructure and enable the delivery and reception of bundles, a "Convergence Layer Adapter" (CLA) is used. The CLA essentially acts as an intermediary between a DTN application and the IP protocol. Different CLA implementations offer varying services to the applications or try to optimize a particular form of communication; the most common examples are the TCP Convergence Layer Protocol [4], Saratoga [10] that mimics UDP and the DTN session layer [5] which is optimized for receiver-driven applications and multicast communication.

As mentioned in the previous section, Voice-over-IP is a technology based on packet-switching that is nowadays standardized and easily accessible. Further, the Session Initiation Protocol is the established standard for setting up VoIP calls and the information it conveys during the call setup process is vital for our application. We are again using the SIP-based Asterisk PBX which is an open source solution offering advanced functionality along with access to the PSTN via special gateways; more importantly, it offers the ability to extend its call handling mechanisms through userdefined programs that can be launched on-demand.

Combining the technologies and tools just described, we built a system that utilizes voicemails to facilitate communication of users residing in isolated and disconnected geographical regions with the "outside" world. More precisely, in regions where communication through live phone calls is not feasible due to different network or capacity limitations, the proposed system records users' messages and attempts to deliver them to their intended recipients in the form of voicemails.

In order to achieve this and allow recorded messages to exit the disconnected geographical regions, the system relies on a Delay Tolerant Network infrastructure for the storage and forwarding of recorded voicemails. Thus, at the application layer, VoIP technology is used to handle the voice messages while a convergence layer is employed to link the two networking interfaces.

From the system architecture (Figure 2), we examine here a couple of major components and operations whereas more details can be found in [7]. 


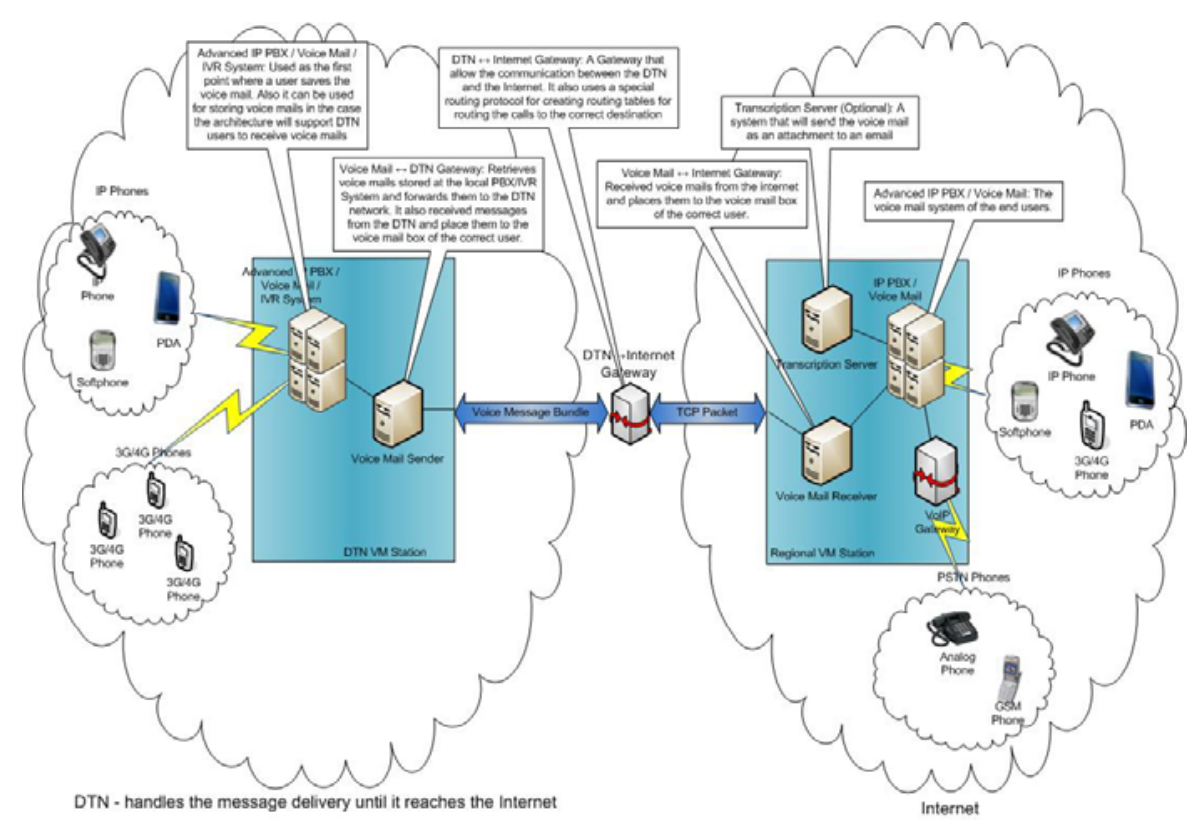

Fig. 2. A DTN architecture for voice messaging

The DTN Voicemail station is in general terms the entity responsible for processing calls originating from users inside a DTN area (i.e., disconnected region), re-cording the voice messages and initiating their delivery procedure. It consists of two modules: the IP-PBX and the Voicemail Sender.

The role of the IP-PBX is to process a user's call request; in the case that connectivity is not available (which should be the norm in a DTN region) instead of dropping the call, it should prompt the user to leave a voice message. After successfully recording the voice message, the PBX creates a special file that contains the actual message along with destination information which can be automatically extracted from the SIP INVITE message.

Once all the required information is in place, the DTN VM Station starts the process of forwarding the recorded voicemail to its intended destination over DTN. The Voicemail Sender is the component responsible for retrieving the voice messages and using their destination information to initiate their delivery. The message recipient can be a SIP user (in the form of a SIP URI) or a number in the PSTN (we refer to this information as Recipient ID).

The next step for the Voicemail Sender is to identify the destination network, i.e., the domain name or the IP of the gateway that serves the recipient. For achieving this, the Voicemail Sender utilizes the DUNDi protocol [6] and more precisely its lookup operation. If the lookup provides the destination domain, the message is sent directly towards that domain; on the other hand, if the lookup operation returns no results, the voice message is blindly forwarded to the DTN - Internet Gateway. Considering that the DTN - Internet Gateway has a permanent connection to the Internet, we assume that it has more updated DUNDi information than the Voicemail Sender (that resides in DTN region). 
Extending the above functionality, the DTN Voicemail station and namely its IPPBX component can act as a repository for incoming voice messages (i.e. for messages that arrive from other DTN regions or the Internet).

In its turn, the DTN-Internet gateway serves two purposes: the first is to provide interoperability between the Delay Tolerant Network and the Internet, and the second is to locate destination users and forward received voicemails to them. To serve the abovementioned purpose the DTN $\leftrightarrow$ Internet Gateway processes bundles which were sent to it by a DTN VM station (namely the Voicemail sender as described above). If the Voicemail sender could not identify the destination do-main, the DUNDi protocol is used again for this purpose. Once the final destination is known, the bundle is converted to TCP/IP packets which are sent over the Internet towards their final destination. The DTN-Internet gateway also maintains a list of potential gateways for PSTN termination; one of these will be used to de-liver the message when the recipient ID is a PSTN number.

At the receiving end of a voice message, a set of components make up the Regional Voicemail station. The first point of contact is the Voicemail Receiver, the entity that receives the voice messages and starts the final delivery process by notifying the associated IP-PBX. According to the recipient-ID a number of actions are possible:

- The recipient is a user of the local IP-PBX (SIP URI); a call is automatically placed to that user's extension and once it is answered the message is played back. If there is no answer, a voicemail is created and deposited in the user's voicemail inbox.

- The recipient is a PSTN number; a call is automatically placed to that number via the gateway connected to the local IP-PBX. Once the call is answered the voice message is played back. If there is no answer the call is retried after some time.

Finally, it is important to emphasize that the proposed system is transparent to the end-users; the caller places a regular phone call leaving a voicemail message and eventually the callee simply receives a regular voicemail message.

\section{Conclusions}

In conclusion, through the two examples presented, we have demonstrated how technologies readily available can be utilized to enhance the telecommunication facilities offered in rural areas. Where coverage has already been extended, community efforts can drive down the cost of access to advanced telecommunications.

Open Access. This article is distributed under the terms of the Creative Commons Attribution Noncommercial License which permits any noncommercial use, distribution, and reproduction in any medium, provided the original author(s) and source are credited.

\section{References}

1. Asterisk - The Open Source Telephony Project, http://www.asterisk.org/ (accessed October 11, 2011)

2. Bundle Protocol Specification, RFC 5050 (2007)

3. Delay Tolerant Networking Architecture, RFC 4838 (2007) 
4. Delay Tolerant Networking TCP Convergence Layer Protocol, Internet Engineering Task Force Draft (2008)

5. Demmer, M., Fall, K.: The Design and Implementation of a Session Layer for DelayTolerant Networks. Computer Communications Journal 32(16), 1724-1730 (2009)

6. Distributed Universal Number Discovery (DUNDi), Internet Draft (2004)

7. Lambrinos, L.: Deploying Open-Source IP telephony in Rural Environments. In: 2nd Next Generation Mobile Applications, Services and Technologies (NGMAST), Cardiff, UK (September 2008)

8. SIP: Session Initiation Protocol, RFC 3261 (2002)

9. Tziouvas, C., Lambrinos, L., Chrysostomou, C.: A delay tolerant platform for voice message delivery. In: International Workshop on Opportunistic and De-lay/DisruptionTolerant Networking (WODTN 2011), Brest, France, October 3-6 (2011)

10. Wood, L., Eddy, W.M., Ivancic, W., McKim, J., Jackson, C.: Saratoga: a Delay-Tolerant Networking Convergence Layer with Efficient Link Utilization. In: International Workshop on Satellite and Space Communications, pp. 168-172 (September 2007) 